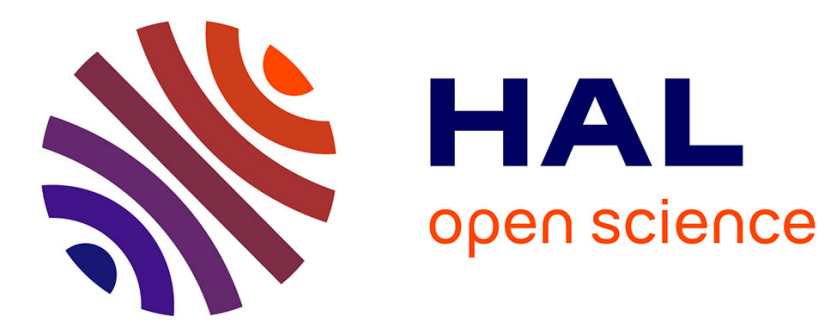

\title{
Settling the APX-Hardness Status for Geometric Set Cover
}

\author{
Nabil Mustafa, Rajiv Raman, Saurabh Ray
}

\section{To cite this version:}

Nabil Mustafa, Rajiv Raman, Saurabh Ray. Settling the APX-Hardness Status for Geometric Set Cover. Proc. of the 55th Annual Symposium on Foundations of Computer Science (FOCS), 2014, Philadelphia, United States. 10.1109/FOCS.2014.64 . hal-01188991

\section{HAL Id: hal-01188991 \\ https://hal.science/hal-01188991}

Submitted on 1 Sep 2015

HAL is a multi-disciplinary open access archive for the deposit and dissemination of scientific research documents, whether they are published or not. The documents may come from teaching and research institutions in France or abroad, or from public or private research centers.
L'archive ouverte pluridisciplinaire HAL, est destinée au dépôt et à la diffusion de documents scientifiques de niveau recherche, publiés ou non, émanant des établissements d'enseignement et de recherche français ou étrangers, des laboratoires publics ou privés. 


\section{Settling the APX-hardness Status for Geometric Set Cover}

\author{
Nabil H. Mustafa \\ Laboratoire d'Informatique Gaspard-Monge, \\ Université Paris-Est, Equipe A3SI, ESIEE Paris. \\ mustafan@esiee.fr
}

\author{
Rajiv Raman \\ Dept. of Computer Science, \\ IIIT, Delhi. \\ rajiv@iiitd.ac.in
}

\author{
Saurabh Ray \\ Computer Science Department, \\ New York University, Abu Dhabi. \\ saurabh.ray@nyu.edu
}

\begin{abstract}
Weighted geometric set-cover problems arise naturally in several geometric and non-geometric settings (e.g. the breakthrough of Bansal and Pruhs (FOCS 2010) reduces a wide class of machine scheduling problems to weighted geometric set-cover). More than two decades of research has succeeded in settling the $(1+\epsilon)$-approximability status for most geometric set-cover problems, except for four basic scenarios which are still lacking. One is that of weighted disks in the plane for which, after a series of papers, Varadarajan (STOC 2010) presented a clever quasi-sampling technique, which together with improvements by Chan et al. (SODA 2012), yielded a $O(1)$-approximation algorithm. Even for the unweighted case, a PTAS for a fundamental class of objects called pseudodisks (which includes disks, unit-height rectangles, translates of convex sets etc.) is currently unknown. Another fundamental case is weighted halfspaces in $\mathbb{R}^{3}$, for which a PTAS is currently lacking. In this paper, we present a QPTAS for all of these remaining problems. Our results are based on the separator framework of Adamaszek and Wiese (FOCS 2013, SODA 2014), who recently obtained a QPTAS for weighted independent set of polygonal regions. This rules out the possibility that these problems are APX-hard, assuming NP $\nsubseteq$ DTIME $\left(2^{\text {polylog(n) }}\right)$. Together with the recent work of Chan-Grant (CGTA 2014), this settles the APX-hardness status for all natural geometric set-cover problems.
\end{abstract}

Keywords-Hitting Sets; Pseudodisks; $k$-admissible regions; Quasi PTAS;

\section{INTRODUCTION}

One of the fundamental optimization problem is the setcover problem: given a range space $(X, \mathcal{R})$ consisting of a set $X$ and a set $\mathcal{R}$ of subsets of $X$ called the ranges, the objective is to compute a minimum-sized subset of $\mathcal{R}$ that covers all the points of $X$. Unfortunately in the general case, it is strongly NP-hard; worse, it is NP-hard to approximate the minimum set-cover within a factor of $c \log n$ of the optimal [24] for some constant $c$.

A natural extensively-studied occurrence of the set-cover problem is when the ranges are derived from geometric objects. For example, given a set $P$ of $n$ points in the plane and a set $\mathcal{R}$ of disks, the set-cover problem for disks asks to compute a minimum cardinality subset of disks whose union covers all the points of $P$. Unfortunately, computing the minimum cardinality set-cover remains NP-hard even for basic geometric objects, such as unit disks in the plane. Effort has therefore been devoted to devising approximation algorithms for geometric set-cover problems (see [3], [8], [7], [6], [14], [21], [4], [9], [5], [20], [10], [23] for a few examples). Nearly all the effort has been for the following natural and fundamental categories of geometric objects: halfspaces, balls (and generally, pseudodisks), axis-parallel rectangles, triangles and objects parameterized by their union-complexity (a set of regions $\mathcal{R}$ has union complexity $\phi(\cdot)$ if the boundary of the union of any $r$ of the regions has at most $r \phi(r)$ intersection points). An important version is the weighted setting, where one seeks to find the minimumweight set-cover.

Research during the past three decades has, in fact, been able to largely answer the question of the existence of a PTAS, or provability of APX-hardness for these problems for the uniform case, where one is minimizing the cardinality of the set-cover. For the more general weighted case, there has been considerable progress recently - an $O(\log \phi(\mathrm{OPT}))$-approximation as a function of the unioncomplexity is possible via the quasi-uniform sampling technique of Varadarajan [26] and its improvement by Chan et al. [5]. On the other hand, recently Chan and Grant [4] proved APX-hardness results for the set-cover problem for a large class of geometric objects. We also point out that for any integer $s$, there exist $O(s)$-sided polygons with union complexity $n 2^{\alpha(n)^{O(s)}}$ for which set-cover is inapproximable within $\Omega(\log s)$. Also, as any set-system with sets of size at most $s$ can be realized by halfspaces in $\mathbb{R}^{2 s}$, an $\Omega(\log d)$ lower-bound follows for approximability of halfspaces setcover in $\mathbb{R}^{d}$. This lower-bound requires $d \geq 4$, leaving open the interesting question of approximation schemes for weighted halfspaces in $\mathbb{R}^{3}$.

See the table for the current status of geometric set-cover. The four open cases present a challenge as the current state-of-the-art methods hit some basic obstacles: the approximation algorithms for weighted halfspaces, balls and pseudodisks use LP-rounding with $\epsilon$-nets [26], [5], and so provably cannot give better than $O(1)$-approximation algorithms. LP rounding was avoided by the use of localsearch technique [20] to give a PTAS for halfspaces $\left(\mathbb{R}^{3}\right)$ and disks $\left(\mathbb{R}^{2}\right)$; however $i$ ) for fundamental reasons it is currently limited to the unweighted case, and $i i$ ) does not extend to pseudodisks. 


\begin{tabular}{|l|c|c|}
\hline Object & Uniform & Weighted \\
\hline Halfspaces $\left(\mathbb{R}^{2}\right)$ & Exact & Exact \\
\hline Halfspaces $\left(\mathbb{R}^{3}\right)$ & PTAS & $?$ \\
\hline Halfspaces $\left(\mathbb{R}^{4}\right)$ & APX-H & APX-H \\
\hline Balls $\left(\mathbb{R}^{2}\right)$ & PTAS & $?$ \\
\hline Pseudodisks $\left(\mathbb{R}^{2}\right)$ & $?$ & $?$ \\
\hline Balls $\left(\mathbb{R}^{3}\right)$ & APX-H & APX-H \\
\hline A-P Rects $\left(\mathbb{R}^{2}\right)$ & APX-H & APX-H \\
\hline A-P Rects $\left(\mathbb{R}^{d}\right)$ & APX-H & APX-H \\
\hline Triangles $\left(\mathbb{R}^{2}\right)$ & APX-H & APX-H \\
\hline Linear U-C $\left(\mathbb{R}^{2}\right)$ & APX-H & APX-H \\
\hline
\end{tabular}

In this paper, we make progress on the approximability status of the remaining four open cases by presenting a quasipolynomial time approximation scheme (QPTAS) for all these problems. This rules out the possibility that these problems are APX-hard, assuming NP $\nsubseteq$ DTIME $\left(2^{\text {polylog }(n)}\right)$. Together with the previous work showing hardness results or PTAS, this settles the APX-hardness status for all natural geometric set-cover problems.

The motivation of our work is the recent progress on approximation algorithms for another fundamental geometric optimization problem, maximum independent sets in the intersection graphs of geometric objects, where $(1+\epsilon)$ approximation algorithms (or even constant factor approximation algorithms) are not known for many objects. In a recent breakthough, Adamaszek and Wiese [1], [2] presented a QPTAS for computing weighted maximum independent set for a variety of geometric objects (e.g., axis-parallel rectangles, line-segments, polygons with polylogarithmically many sides) in the plane (the algorithm runs in time $2^{p o l y(\log n / \epsilon)}$ ). We now sketch their main idea for approximating the maximum independent set for weighted line-segments in the plane, for which let OPT be the optimal solution. The key tool is the existence of a closed polygonal curve $\mathcal{C}$ (with few vertices) that intersects segments in OPT with small total weight, and at least a constant fraction of the total weight of OPT lies in the two regions created by $\mathcal{C}$. Hence one can guess the curve $\mathcal{C}$ (which does not require knowing ОРт) ${ }^{1}$, and then return the union of solution of the two subproblems (which are solved recursively). With appropriate parameters, the loss incurred by throwing away the segments intersecting $\mathcal{C}$ is at most $\epsilon$-th fraction of the optimal solution, yielding a $(1-\epsilon)$-approximation in quasi-polynomial time.

Let us consider how the above technique can be made to work for the set cover problem. Assume that we are given a set $P$ of $n$ points and a set $\mathcal{R}$ of $m$ weighted disks and our goal is to pick a minimum-weight set-cover from $\mathcal{R}$. We can again consider the optimal solution OPT and hope to find a curve $\mathcal{C}$ which intersects objects in OPT with small total weight and has a constant fraction of the weight of

\footnotetext{
${ }^{1}$ The guessing is actually done by enumerating all possible curves. The fact that $\mathcal{C}$ has a small number of vertices allows efficient enumeration.
}

OPT in the interior as well as the exterior. However such a curve does not always exist - consider, e.g., a case where the optimal solution consists of a set of disks that share a common point (not necessarily in $P$ ). Crucially, unlike the independent set problem, the objects in the optimal set-cover are not disjoint. This dooms any separator-based approaches for the set-cover problem.

Surprisingly, we show that nevertheless there still exists a curve $\mathcal{C}$ (which may, in fact, intersect all the disks in $\mathcal{R}$ !) such that solving the induced sub-problems in the interior and exterior of $\mathcal{C}$ and combining them leads to a near-optimal solution (Theorem III.1). The problem is further complicated by arbitrary weights on the disks. As a result, several promising approaches (including the quasisampling technique of Varadarajan [26]) fail. Fortunately, generalizing the problem to pseudodisks and then using structural properties of pseudodisks melded with randomized ordering and probabilistic re-sampling techniques works out. Such separator based techniques do not work in three dimensions (even for the independent set problem, one can show that there exists a set of disjoint segments in $\mathbb{R}^{3}$ so that there is no compactly-represented polyhedral separator). In fact, even for unit balls in $\mathbb{R}^{3}$ all containing a common point, the set cover problem is APX-hard [4]! However, when the objects are halfspaces in $\mathbb{R}^{3}$, we prove the existence of a polyhedral separator that allows us to get a QPTAS. This shows that the set-cover problem for halfspaces is the only natural problem in $\mathbb{R}^{3}$ that is not APX-hard.

We refer to the full version of this paper [19] for missing proofs.

\section{Preliminaries}

Let $\mathcal{R}=\left\{R_{1}, \ldots, R_{n}\right\}$ be a set of weighted $\alpha$-simple regions in the plane, where a bounded and connected region in the plane is called $\alpha$-simple if its boundary can be decomposed into at most $\alpha x$-monotone $\operatorname{arcs}^{2}$. For any $\alpha$-simple region $R$, we denote by $\Gamma(R)$ a set of at most $\alpha$ $x$-monotone curves that its boundary can be decomposed into. For a set $\mathcal{R}$ of $\alpha$-simple regions, we define $\Gamma(\mathcal{R})$ to be the set $\bigcup_{R \in \mathcal{R}} \Gamma(R)$. Let $w_{i}$ denote the weight of the region $R_{i} \in \mathcal{R}$, and $w(\mathcal{S})$ be the total weight of the regions in $\mathcal{S}$ (set $W=w(\mathcal{R})$ ). The regions in $\mathcal{R}$ need not be disjoint ${ }^{3}$. A collection of compact simply connected regions in the plane is said to form a family of pseudodisks if the boundaries of any two of the regions intersect at most twice. The union complexity of a set of pseudodisks in linear [22]. For technical reasons we will assume that pseudodisks in this paper are $\alpha$-simple for some constant $\alpha$. This restriction is not crucial, and can be removed [12]. A

\footnotetext{
${ }^{2}$ Note that whether a region is $\alpha$-simple depends on choice of axes.

${ }^{3}$ We assume $\mathcal{R}$ to be in general position, so no three regions boundaries intersect at the same point.
} 
collection of regions $\mathcal{R}$ is said to be cover-free if no region $R \in \mathcal{R}$ is covered by the union of the regions in $\mathcal{R} \backslash R$. For any closed Jordan curve $\mathcal{C}$, we denote the closed region bounded by it as interior $(\mathcal{C})$ and the closed unbounded region defined by it as exterior $(\mathcal{C})$. Given $\mathcal{R}$, we denote by $\mathcal{R}_{i n}(\mathcal{C})\left(\mathcal{R}_{\text {ext }}(\mathcal{C})\right)$ the subset of the regions that lie in interior $(\mathcal{C})($ exterior $(\mathcal{C}))$. Similarly if $P$ is a set of points, we denote by $P_{i n}(\mathcal{C})\left(P_{e x t}(\mathcal{C})\right)$ the subset of points lying in interior $(\mathcal{C})($ exterior $(\mathcal{C}))$.

VC-dimension and $\epsilon$-nets [15]. Given a range space $(X, \mathcal{F})$, a set $X^{\prime} \subseteq X$ is shattered if every subset of $X^{\prime}$ can be obtained by intersecting $X^{\prime}$ with a member of the family $\mathcal{F}$. The VC-dimension of $(X, \mathcal{F})$ is the size of the largest set that can be shattered. Given a set system $(X, \mathcal{F})$ where each element of $X$ has a positive weight associated with it, and a paramter $0<\epsilon<1$, an $\epsilon$-net is a subset $Y \subseteq X$ s.t. for any $F \in \mathcal{F}$ with weight at least an $\epsilon$ fraction of the total weight, $Y \cap F \neq \emptyset$. The $\epsilon$-net theorem (Haussler-Welzl [13]) states that there exists an $\epsilon$-net of size $O(d / \epsilon \log 1 / \epsilon)$ for any range space with VC-dimension $d$.

QPT-partitionable problems. Given an optimization problem $\mathcal{O}$, let $\operatorname{OPT}_{\mathcal{O}}(I)$ denote the optimal solution of $\mathcal{O}$ on the instance $I$, and let $w\left(\operatorname{OPT}_{\mathcal{O}}(I)\right)$ be the weight of this optimal solution. We assume $\mathcal{O}$ is a minimization problem; similar statements hold for the maximization case.

Definition II.1. A problem $\mathcal{O}$ is quasi-polynomial time partitionable (QPT-partitionable) if, given any input I and a parameter $\delta>0$, there exist a constant $c<1, k=$ $O\left(n^{(\log n / \delta)^{O(1)}}\right)$, and instance pairs $\left(I_{l}^{1}, I_{r}^{1}\right), \ldots,\left(I_{l}^{k}, I_{r}^{k}\right)$ (computable in time polynomial in $k$ ), and an index $j, 1 \leq$ $j \leq k$, such that $i) \max \left\{w\left(\operatorname{OPT}_{\mathcal{O}}\left(I_{l}^{j}\right)\right), w\left(\operatorname{OPT}_{\mathcal{O}}\left(I_{r}^{j}\right)\right)\right\} \leq$ $c \cdot w\left(\mathrm{OPT}_{\mathcal{O}}(I)\right)$, ii $) \operatorname{OPT}_{\mathcal{O}}\left(I_{l}^{j}\right) \cup \mathrm{OPT}_{\mathcal{O}}\left(I_{r}^{j}\right)$ is a feasible solution, and iii) $w\left(\operatorname{OPT}_{\mathcal{O}}\left(I_{l}^{j}\right)\right)+w\left(\operatorname{OPT}_{\mathcal{O}}\left(I_{r}^{j}\right)\right) \leq(1+$ $\delta) w\left(\operatorname{OPT}_{\mathcal{O}}(I)\right)$.

The next lemma follows immediately from recursive divideand-conquer:

Lemma II.1. If a problem $\mathcal{O}$ is QPT-partitionable, and if for any instance $I, w\left(\operatorname{OPT}_{\mathcal{O}}(I)\right) \geq 1$, then one can compute a $(1+\epsilon)$-approximate solution for $\mathcal{O}$ in time $O\left(n^{\left(\frac{1}{\epsilon} \cdot \log w\left(\operatorname{OPT}_{\mathcal{O}}(I)\right) \cdot \log n\right)^{O(1)}}\right)$.

Proof: The algorithm will return an approximate solution $\operatorname{APPROX}_{\mathcal{O}}(I)$ as follows. Let $T=w\left(\operatorname{OPT}_{\mathcal{O}}(I)\right)$, and set $\delta=\Theta(\epsilon / \log T)$. Construct the $k$ instance pairs $\left(I_{l}^{1}, I_{r}^{1}\right), \ldots,\left(I_{l}^{k}, I_{r}^{k}\right)$, where $k=O\left(n^{(1 / \epsilon \cdot \log T \cdot \log n)^{O(1)}}\right)$. For each $i=1 \ldots k$, compute $\operatorname{APPROX}_{\mathcal{O}}\left(I_{l}^{i}\right)$ and $\operatorname{APPROX}_{\mathcal{O}}\left(I_{r}^{i}\right)$ recursively and return the solution $\operatorname{APPROX}_{\mathcal{O}}\left(I_{l}^{j}\right) \cup \operatorname{APPROX}_{\mathcal{O}}\left(I_{r}^{j}\right), \quad$ where $j=\arg \min _{i} w\left(\operatorname{APPROX}_{\mathcal{O}}\left(I_{l}^{i}\right)\right)+w\left(\operatorname{APPROX}_{\mathcal{O}}\left(I_{r}^{i}\right)\right)$.
We can prune the recursion tree at the level $l=O(\log T)$ since for the right choice of $i$ at each recursion, the weight of the optimal solution falls by a constant factor with every recursive call. The size of the tree is at most $(2 k)^{l}=O\left(n^{(1 / \epsilon \cdot \log T \cdot \log n)^{O(1)}}\right)$. It can be shown inductively that the approximation factor of a sub-problem $t$ levels away from the lowest level is $(1+\delta)^{t}$. Thus the approximation factor at the root is $(1+\delta)^{l} \leq(1+\epsilon)$, with appropriate constants in the definition of $\delta$. The time taken by the algorithm is $O\left(n^{(1 / \epsilon \cdot \log T \cdot \log n)^{O(1)}}\right)$.

Geometric separators.: A $\delta$-separator for $\mathcal{R}$, given $\delta>0$, is a simple closed curve $\mathcal{C}$ in the plane such that the weight of the regions of $\mathcal{R}$ completely inside (and outside) $\mathcal{C}$ is at most $2 W / 3$ (such a curve is called balanced), and the total weight of the regions in $\mathcal{R}$ intersecting $\mathcal{C}$ is at most $\delta W$. The goal is to show the existence, given $\mathcal{R}$ and $\delta>0$, of separators of small combinatorial complexity as a function of $n, m$ (number of intersections in $\mathcal{R}$ ), $\alpha$ and $\delta$. The existence of small $\delta$-separators was the core of the result of [1]; later it was noted independently by MustafaRaman-Ray (see [18]), Adamaszek and Wiese [2] and HarPeled [12] that the construction in [1] can be made optimal using the techniques of constructing cuttings and $\epsilon$-nets (i.e, the probabilistic re-sampling technique) [15].

We state two separator results that we will be using in our algorithm.

Theorem II.2 ( [18], [12]). Given a set $\mathcal{R}$ of $n$ weighted $\alpha$-simple regions (with total weight $W$, and no curve having weight more than $W / 3$ ) with disjoint interiors, and a parameter $\delta>0$, there exists a simple closed curve $\mathcal{C}$ such that $i)$ the total weight of the regions intersecting $\mathcal{C}$ is at most $\delta W$, and $i$ ) the total weight of the regions completely inside or outside $\mathcal{C}$ is at most $2 \mathrm{~W} / 3$. Furthermore the complexity of $\mathcal{C}$ is $T=O(\alpha / \delta)$. That is $\mathcal{C}$ can be completely described by a sequence of at most $T$ curves of $\Gamma(\mathcal{R})$ and additional at most $T$ bits. Furthermore this is optimal; even when $\mathcal{R}$ is set of disjoint line segments, any $\mathcal{C}$ satisfying these two properties must have $\Omega(1 / \delta)$ bends.

In the case when the regions have uniform weights (say each region has weight one) but are not necessarily disjoint:

Theorem II.3 ( [18]). Given a set $\mathcal{R}$ of $n$-simple regions in the plane with $m$ intersections, and a parameter $r$, there exists a simple closed curve $\mathcal{C}$ such that i) the number of regions in $\mathcal{R}$ intersecting $\mathcal{C}$ are $O\left(\left(m+\frac{\alpha^{2} n^{2}}{r}\right)^{1 / 2}\right)$, and ii) the total number of regions completely inside or outside $\mathcal{C}$ is at most $2 n / 3$. Furthermore, complexity of $\mathcal{C}$ is $T=O\left(\left(r+\frac{m r^{2}}{\alpha^{2} n^{2}}\right)^{1 / 2}\right)$. That is, $\mathcal{C}$ can be completely described by a sequence of at most $T$ curves of $\Gamma(\mathcal{R})$ and at most $T$ additional bits.

The technical condition that the regions are $\alpha$-simple in the 
theorems above can be removed [12].

Geometric Set Cover: Let $\mathcal{R}=\left\{R_{1}, \ldots, R_{n}\right\}$ be a set of weighted regions (in $\mathbb{R}^{2}$ or $\mathbb{R}^{3}$ ) and let $P$ be a finite set of points in the plane. The goal is to compute a subset $\mathcal{Q} \subseteq \mathcal{R}$ minimizing the total weight $w(\mathcal{Q})$ so that $P \subseteq \cup_{Q \in \mathcal{Q}} Q$. We will denote an optimal solution $\mathcal{Q}$ for an instance of the problem given by a set of regions $\mathcal{R}$ and a set of points $P$ by $\operatorname{Opt}(\mathcal{R}, P)$, and its weight by $w(\operatorname{Opt}(\mathcal{R}, P))$.

Claim II.4. If there exists a QPTAS for set-systems $\left(\mathcal{R}^{\prime}, P^{\prime}\right)$ where $i$ ) each $R \in \mathcal{R}^{\prime}$ has weight $w(R) \geq 1$, and ii) the weight of the optimal set-cover for $\left(\mathcal{R}^{\prime}, P^{\prime}\right)$ is $O(n / \epsilon)$, then there exists a QPTAS for the minimum-weight set cover for a set-system $(\mathcal{R}, P)$ with arbitrary weights.

Proof: Let $\mathcal{Q}$ be a minimum-weight set-cover for $(\mathcal{R}, P)$. First guess the maximum weight region in $\mathcal{Q}$, say of weight $w_{\max }$ (there are $n$ such choices). Then by exponential search on the interval $\left[w_{\max }, n w_{\max }\right]$, one can guess the weight of $\mathcal{Q}$ within a $(1+\epsilon / 3)$ factor (there are $O\left(\log _{1+\epsilon} n\right)$ such choices). Let $w_{\text {aprx }}$ be this weight, satisfying $w(\mathcal{Q}) \leq w_{\text {apr } x} \leq(1+\epsilon / 3) w(\mathcal{Q})$. Set $\mathcal{R}^{\prime} \subset \mathcal{R}$ to be the set of regions with weight at least $\epsilon w_{\text {apr } x} / n$, and $\mathcal{R}^{\prime \prime}=\mathcal{R} \backslash \mathcal{R}^{\prime}$. Let $P^{\prime} \subseteq P$ be the set of points not covered by $\mathcal{R}^{\prime \prime}$, and construct a $(1+\epsilon / 3)$ approximate set-cover $\mathcal{Q}^{\prime}$ to $\left(\mathcal{R}^{\prime}, P^{\prime}\right)$. Return $\mathcal{Q}^{\prime} \cup \mathcal{R}^{\prime \prime}$ as a set-cover for $(\mathcal{R}, P)$. Note that this is the required approximation:

$$
\begin{aligned}
w\left(\mathcal{Q}^{\prime} \cup \mathcal{R}^{\prime \prime}\right) & =w\left(\mathcal{Q}^{\prime}\right)+w\left(\mathcal{R}^{\prime \prime}\right) \\
& \leq(1+\epsilon / 3) w\left(\operatorname{OPT}\left(\mathcal{R}^{\prime}, P^{\prime}\right)\right)+\epsilon w_{\text {apr } x} \\
& \leq(1+\epsilon / 3) w(\mathcal{Q})+\epsilon(1+\epsilon / 3) w(\mathcal{Q}) \\
& \leq(1+\epsilon) w(\mathcal{Q})
\end{aligned}
$$

Above we use the fact that $\mathcal{Q}$ is also a set-cover for $\left(\mathcal{R}^{\prime}, P^{\prime}\right)$. Scaling by $n / \epsilon w_{\text {apr } x}$, each set in $\mathcal{R}^{\prime}$ has weight at least 1 , and weight of $\operatorname{OPT}\left(\mathcal{R}^{\prime}, P^{\prime}\right)=O(n / \epsilon)$.

Hence for the purpose of a $(1+\epsilon)$-approximation, we can assume that the minimum weight of any region is 1 and the weight of the optimal set-cover is $O(n / \epsilon)$.

\section{QPTAS FOR WEIGHTED PSEUDODISKS IN $\mathbb{R}^{2}$}

Our main result in this section is:

Theorem III.1. Let $\mathcal{R}=\left\{R_{1}, \ldots, R_{n}\right\}$ be a set of $n$ weighted $\alpha$-simple pseudodisks with minimum weight 1 . Let $P$ be a set of points in the plane, with no point lying on the boundary of any of the pseudodisks. Assume also that no pseudodisk in $\operatorname{OPT}(\mathcal{R}, P)$ has weight more than $w(\operatorname{Opt}(\mathcal{R}, P)) / 3$. Then for any $\delta>0$, there exists a curve $\mathcal{C}$ such that

- $w\left(\operatorname{Opt}\left(\mathcal{R}, P_{i n}(\mathcal{C})\right)\right) \leq\left(\frac{2}{3}+3 \delta\right) w(\operatorname{Opt}(\mathcal{R}, P))$

- $w\left(\operatorname{Opt}\left(\mathcal{R}, P_{\text {ext }}(\mathcal{C})\right)\right) \leq\left(\frac{2}{3}+3 \delta\right) w(\operatorname{Opt}(\mathcal{R}, P))$

- $w\left(\operatorname{Opt}\left(\mathcal{R}, P_{i n}(\mathcal{C})\right)\right)+w\left(\operatorname{Opt}\left(\mathcal{R}, P_{\text {ext }}(\mathcal{C})\right)\right)$

$$
\leq(1+2 \delta) w(\operatorname{Opt}(\mathcal{R}, P))
$$

- The complexity of $\mathcal{C}$ is $O\left(\frac{\alpha}{\delta^{2}} \log w(\mathrm{OPT}(\mathcal{R}, P))\right.$.

A QPTAS for weighted pseudodisks follows from this theorem in similar manner to that of Adamaszek and Wiese [1], [2]. We first use Claim II.4 to reduce the given instance of the set cover problem to an instance $(\mathcal{R}, P)$ where the minimum weight of the regions is 1 and the weight of the optimal solution $w(\operatorname{OPT}(\mathcal{R}, P))$ is $O(n / \epsilon)$. Assume also that no pseudodisk in $\operatorname{OPT}(\mathcal{R}, P)$ has weight more than $w(\operatorname{OPT}(\mathcal{R}, P)) / 3$. The input instance can be easily perturbed so that no point lies on the boundary of any region. Now by applying Theorem III.1 with a given $\delta$, there exists a curve $\mathcal{C}$ of complexity $O\left(1 / \delta^{2} \log (n / \epsilon)\right)$. Thus by enumeration, there are $O\left(n^{\left(1 / \delta^{2} \cdot \log n / \epsilon\right)^{O(1)}}\right)$ such possible curves $\mathcal{C}^{\prime}$ (the proof of Theorem III.1 shows that the vertices of any such $\mathcal{C}^{\prime}$ come from a polynomial-sized subset that can be computed in polynomial time) each giving two sub-problems $\left(\mathcal{R}, P_{\text {in }}\left(\mathcal{C}^{\prime}\right)\right)$ and $\left(\mathcal{R}, P_{\text {ext }}\left(\mathcal{C}^{\prime}\right)\right)$. Thus, as $\epsilon$ is a constant, the problem is QPT-partitionable, which together with Lemma II.1 gives the required QPTAS. Finally, note that there can be at most 2 pseudodisks in the optimal solution with weight more than $w(\mathrm{OPT}(\mathcal{R}, P)) / 3$, and one can simply guess (by enumerating the at most $O\left(n^{2}\right)$ possibilities) these pseudodisks, and recurse on the subproblem where the weight of the optimal solution is reduced by a constant factor.

Towards proving the above theorem, we define structural decompositions for pseudodisks in $\mathbb{R}^{2}$ called core decompositions. We will also use this notion for halfspaces in $\mathbb{R}^{3}$. Informally, given a set of pseudodisks, our goal is to shrink them in such a way that their union remains (almost) unchanged but the number of vertices ${ }^{4}$ in the arrangement decreases. Denote by $B_{\tau}$ a closed ball of radius $\tau$. We denote the Minkowski sum by $\oplus$.

Definition III.2 (Core Decomposition.) Given $\mathcal{R}=$ $\left\{R_{1}, \ldots, R_{n}\right\}$ and a $\beta>0$, a set of regions $\tilde{\mathcal{R}}=$ $\left\{\tilde{R}_{1}, \ldots, \tilde{R}_{n}\right\}$ is called a $\beta$-core decomposition of $\mathcal{R}$ (and each $\tilde{R}_{i}$ a core of $R_{i}$ ) if (1) $\tilde{R}_{i} \subseteq R_{i}$ for all $i=1, \ldots, n,(2)$ $\bigcup_{i} \tilde{R}_{i} \supseteq \bigcup_{i} R_{i} \backslash \bigcup_{i}\left(\partial R_{i} \oplus B_{\beta}\right)$, and (3) each $\tilde{R}_{i}$ is simply connected.

Each disk $\tilde{R}_{i} \in \tilde{\mathcal{R}}$ will be composed of pieces of boundaries of the disks in $\mathcal{R}$. The sequence of the endpoints of these pieces will be the vertices defining $\tilde{R}_{i}$ (denote this sequence by $v\left(\tilde{R}_{i}\right)$, and its cardinality by $\left.\left|v\left(\tilde{R}_{i}\right)\right|\right)$. The information needed to uniquely determine $\tilde{R}_{i}$ then is the sequence of these pieces, or equivalently, the sequence of vertices defining $\tilde{R}_{i}$. In the following, we will use the term core decomposition to mean a $\beta$-core decomposition with a suitably small $\beta>0$ to be fixed later. The following

\footnotetext{
${ }^{4}$ By vertices we mean the intersection points of the boundaries of the pseudodisks.
} 


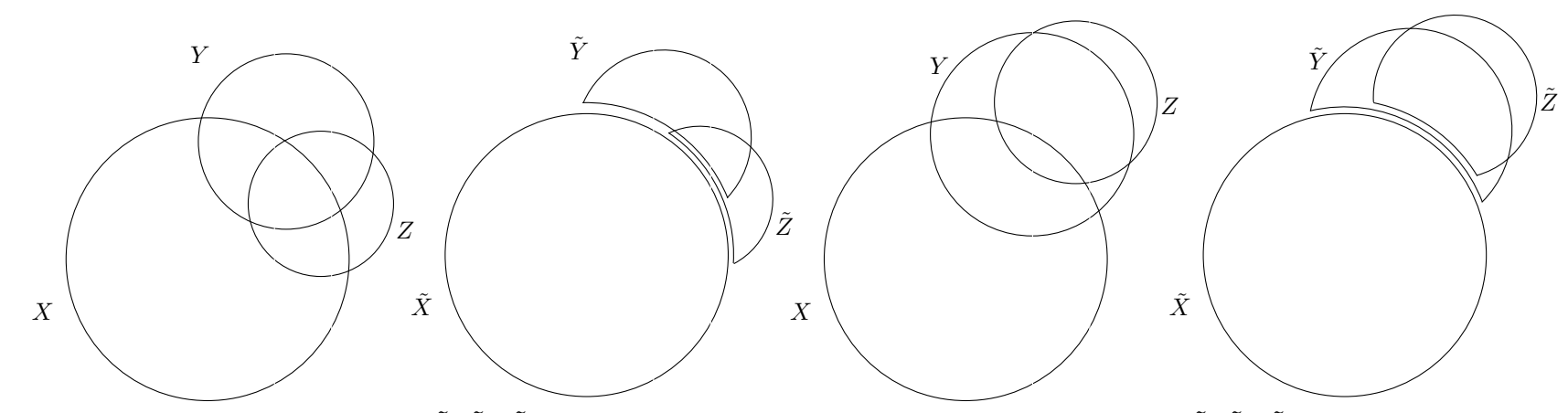

(a) $X, Y, Z$

(b) $\tilde{X}, \tilde{Y}, \tilde{Z}$

(c) $X, Y$ and $Z$

(d) $\tilde{X}, \tilde{Y}, \tilde{Z}$

Figure 1: Pushing pseudodisks

two lemmas show the existence of core decompositions with specific properties.

Lemma III.3. Given a cover-free set $\mathcal{R}$ of pseudodisks, a marked pseudodisk $X \in \mathcal{R}$ (called the pusher) and $a \beta>0$, there exists a $\beta$-core decomposition $\tilde{\mathcal{R}}$ of $\mathcal{R}$ such that $\tilde{X}=$ $X$ and $\tilde{R} \cap \tilde{X}=\emptyset$ for all $R \neq X$ and $\{\tilde{R}: R \in \mathcal{R}\}$ is a cover-free family of pseudodisks.

Proof: Set $\tilde{X}=X$. For each $R \in \mathcal{R} \backslash\{X\}$, we compute a number $\operatorname{gap}(R) \in(0, \mu)$, where $\mu \in(0, \beta)$ is a suitably small number, and set $\tilde{R}=\operatorname{closure}\left(R \backslash\left(X \oplus B_{\text {gap }(R)}\right)\right)$ (we say that $X$ pushes $R$ with gap $\operatorname{gap}(R)$ ). For any $R \in$ $\mathcal{R} \backslash\{X\}$, let $I_{R}$ be the interval $R \cap \partial X$ on the boundary of $X$. As no pseudodisk in $\mathcal{R}$ is completely contained in any other pseudodisk of $\mathcal{R}$ (cover-free), the intervals $I_{R}$ are well-defined. Consider the partial order $\preceq$ on these intervals defined by inclusion $\left(I_{R} \preceq I_{S}\right.$ if $\left.I_{R} \subset I_{S}\right)$. By a topological sorting of this partial order we can assign a distinct rank $\operatorname{rank}(R) \in\{1, \ldots, n\}$ to each pseudodisk $R \in \mathcal{R}$ such that if $I_{R} \subset I_{R^{\prime}}$ then $\operatorname{rank}(R)>\operatorname{rank}\left(R^{\prime}\right)$. We set $\operatorname{gap}(R)=$ $\mu \frac{\operatorname{rank}(R)}{n}$.

Clearly each core is contained in its corresponding pseudodisk and for a small-enough $\mu$, it is simply connected. Also, since the points we may have removed from the union, due to the gaps, lie in $X \oplus B_{\mu}$, the second condition in the definition of a $\beta$-core decomposition is satisfied. The cores obtained are also cover-free because the union of cores cover the union of the original regions (except close to boundaries). Since the input set is cover-free each pseudodisk has a free portion that is not covered by others. The core corresponding to a pseudodisk then must cover the free portion in that pseudodisk which is not covered by the other cores. Thus no core is covered by the union of other cores.

It remains to show that the cores form pseudodisks. Let $Y$ and $Z$ be any two pseudodisks in $\mathcal{R}$, and we now finish the proof by showing that the boundaries of $\tilde{Y}$ and $\tilde{Z}$ intersect at most twice. The possible cases are the following: (1) $I_{Y} \cap$
$I_{Z}=\emptyset$, (2) $I_{Y} \preceq I_{Z}$ and (3) $I_{Y} \cap I_{Z} \neq \emptyset$ and $I_{Y} \npreceq I_{Z}$.

In case 1 , since the intervals are disjoint, $\partial \tilde{Y}$ and $\partial \tilde{Z}$ do not have any new intersection that $\partial Y$ and $\partial Z$ did not have. They may have lost intersections lying in $X$. In any case, $\partial \tilde{Y}$ and $\partial \tilde{Z}$ intersect at most twice. In case $2, Y$ gets pushed with a smaller gap than $Z$ and the situation is exactly as shown in Figures $1 \mathrm{c}$ and $1 \mathrm{~d}$. In case $3, Y$ and $Z$ get pushed with different gaps and the situation is exactly as shown in Figures $1 \mathrm{a}$ and $1 \mathrm{~b}$.

Remark: Note that for each pseudodisk $R$ intersecting $X$, the boundary of $\tilde{R}$ now has two new vertices corresponding to the two intersections of $\partial R$ with $\partial X$. These vertices are slightly perturbed (and arbitrarily close) from the intersections because of the gap. We say that each such new vertex corresponds to the original intersection vertex between $\partial R$ and $\partial X$. When the context is clear, we will not distinguish between this new vertex and the vertex it corresponds to.

Before we prove our next main result on core decompositions, we will need the following technical result. For clarity, vertex $v$, the intersection point of $R_{i}$ and $R_{j}$, is written as $(v, i, j)$. Given $\mathcal{R}$, the depth of a vertex $(v, i, j)$, denoted $d_{v}$, is the total weight of the regions in $\mathcal{R}$ containing $(v, i, j)$ in the interior (thus it excludes the weight of $R_{i}$ and $R_{j}$ ).

Claim III.4. Let $\mathcal{R}=\left\{R_{1}, \ldots, R_{n}\right\}$ be a set of $n$ weighted pseudodisks, and $k>0$ a given parameter. Assume a region $R_{i}$ has weight $w_{i}$, and $W=\sum_{i} w_{i}$. Then

$$
\sum_{\substack{(v, i, j) s . t . \\ k \leq d_{v}<2 k}} \frac{w_{i} \cdot w_{j}}{w_{i}+w_{j}+k}=O(W)
$$

Proof: For the unweighted case, this is easier to derive from the following observation: by the Clarkson-Shor technique, the number of vertices of depth roughly $k$ is $O(n k)$, and each such vertex contributes to the boundary complexity of $O(k)$ regions with probability $O\left(1 / k^{2}\right)$.

We present the complete proof for the weighted case now, 
which follows from melding the Clarkson-Shor technique with a charging argument. Let $\mathcal{R}_{1} \subset \mathcal{R}$ be the set of disks with weight $w_{i} \geq 2 k$, and $\mathcal{R}_{2}=\mathcal{R} \backslash \mathcal{R}_{1}$. Note that $\left|\mathcal{R}_{1}\right|=$ $O(W / k)$, and that any vertex $v$ with depth less than $2 k$ and defined by two disks in $\mathcal{R}_{1}$ must lie on the boundary of the union of the regions in $\mathcal{R}_{1}$. This implies

$$
\begin{aligned}
\sum_{\substack{(v, i, j) \text { s.t. } \\
R_{i}, R_{j} \in \mathcal{R}_{1} \\
k \leq d_{v}<2 k}} \frac{w_{i} \cdot w_{j}}{w_{i}+w_{j}+k} \leq \sum_{\substack{(v, i, j) \text { s.t. } \\
R_{i}, R_{j} \in \mathcal{R}_{1} \\
k \leq d_{v}<2 k}} \min \left\{w_{i}, w_{j}\right\} \\
=O\left(\sum_{R_{i} \in \mathcal{R}_{1}} w_{i}\right)=O(W)
\end{aligned}
$$

where the second-last inequality follows from a charging argument: the number of vertices in the union of $t$ pseudodisks is $O(t)$ [22], and so each vertex can be assigned to one of its two disks such that each disk gets $O(1)$ assigned vertices.

Set $S=\mathcal{R}_{1}$, and further add each disk $R_{i} \in \mathcal{R}_{2}$ into $S$ with probability $p_{i}=w_{i} / 4 k$. Then the expected union complexity of $S$ is:

$$
E[O(|S|)]=O\left(\left|\mathcal{R}_{1}\right|+\sum_{R_{i} \in \mathcal{R}_{2}} \frac{w_{i}}{4 k}\right)=O(W / k)
$$

On the other hand, the expected number of vertices defined by the intersection of a disk in $\mathcal{R}_{2}$ and a disk in $\mathcal{R}_{1}$, and of depth less than $2 k$, that end up as vertices in the union of $S$ :

$$
\begin{array}{r}
\sum_{\substack{(v, i, j) \text { s.t.t } \\
k \leq d_{v} \in \mathcal{R}_{2}, R_{j} \in \mathcal{R}_{1}}} \frac{w_{i}}{4 k} \prod_{\substack{R_{l} \text { contains } v \\
R_{l} \in \mathcal{R}_{2}}}\left(1-p_{l}\right) \\
\geq \sum \frac{w_{i}}{4 k} e^{-2 \sum_{l} w_{l} / 4 k} \geq \sum \frac{w_{i}}{4 e k}
\end{array}
$$

using the fact that $1-x \geq e^{-2 x}$ for $0 \leq x \leq 0.5$, and that $\sum w_{l} \leq 2 k$ as all such $R_{l}$ contain $v$, which has depth at most $2 k$. The expected number of vertices in the union of $S$ defined by two disks in $\mathcal{R}_{2}$, and of depth at most $2 k$ :

$$
\sum_{\substack{(v, i, j) \text { s.t. } \\ R_{i} \in \mathcal{R}_{2}, R_{j} \in \mathcal{R}_{2} \\ k \leq d_{v}<2 k}} \frac{w_{i}}{4 k} \cdot \frac{w_{j}}{4 k} \prod_{\substack{R_{l} \text { contains } v \\ R_{l} \in \mathcal{R}_{2}}}\left(1-p_{l}\right) \geq \sum \frac{w_{i} \cdot w_{j}}{16 e k^{2}}
$$

Putting the lower- and upper-bounds together, we arrive at:

$$
\sum_{\substack{(v, i, j) \text { s.t. } \\ R_{i} \in \mathcal{R}_{2}, R_{j} \in \mathcal{R}_{1}}} \frac{w_{i}}{4 e k}+\sum_{\substack{(v, i, j) \text { s.t. } \\ R_{i} \in \mathcal{R}_{2}, R_{j} \in \mathcal{R}_{2}}} \frac{w_{i} \cdot w_{j}}{16 e k^{2}} \leq O(W / k)
$$

Finally,

$$
\begin{aligned}
& \sum_{\substack{(v, i, j) \text { s.t. } \\
k \leq d_{v}<2 k}} \frac{w_{i} \cdot w_{j}}{w_{i}+w_{j}+k}=\sum_{\substack{(v, i, j) \\
R_{i} \in \mathcal{R}_{2}, R_{j} \in \mathcal{R}_{1}}} \frac{w_{i} \cdot w_{j}}{w_{i}+w_{j}+k} \\
& +\sum_{\substack{(v, i, j) \\
R_{i} \in \mathcal{R}_{2}, R_{j} \in \mathcal{R}_{2}}} \frac{w_{i} \cdot w_{j}}{w_{i}+w_{j}+k} \\
& +\sum_{\substack{(v, i, j) \\
R_{i} \in \mathcal{R}_{1}, R_{j} \in \mathcal{R}_{1}}} \frac{w_{i} \cdot w_{j}}{w_{i}+w_{j}+k} \\
& \leq \sum_{\substack{(v, i, j) \\
R_{i} \in \mathcal{R}_{2}, R_{j} \in \mathcal{R}_{1}}} w_{i} \\
& +\sum_{\substack{(v, i, j) \\
R_{i} \in \mathcal{R}_{2}, R_{j} \in \mathcal{R}_{2}}} \frac{w_{i} \cdot w_{j}}{k}+O(W) \\
& =O(W)
\end{aligned}
$$

where the last inequality follows from Equation (2).

Lemma III.5. Let $\mathcal{R}=\left\{R_{1}, \ldots, R_{n}\right\}$ be a set of $n$ weighted pseudodisks, where $R_{i}$ has weight $w_{i}$, and $W=$ $\sum_{i} w_{i}$. Then there exists a core decomposition of $\mathcal{R}$, say the set $\tilde{\mathcal{R}}=\left\{\tilde{R}_{1}, \ldots, \tilde{R}_{n}\right\}$, such that the pseudodisks in $\tilde{\mathcal{R}}$ are pairwise disjoint, and

$$
\sum_{i}\left|v\left(\tilde{R}_{i}\right)\right| \cdot w_{i}=O(W \log W)
$$

Proof: Recall that $v\left(\tilde{R}_{i}\right)$ is the sequence of vertices defining $\tilde{R}_{i}$ (which will be composed of pieces of boundaries of regions in $\mathcal{R}$ ), and determines $\tilde{R}_{i}$.

The algorithm to construct $\tilde{\mathcal{R}}$ is the following. Construct a permutation, say $\pi$, of $\mathcal{R}$ randomly w.r.t. to the weight distribution of the disks as follows. Pick a random disk, where $R_{i}$ is picked with probability $w_{i} / W$. Set this disk to be the first disk in the permutation, and recursively construct the rest of the permutation on the remaining disks. Let $\pi_{i}$ denote the position of $R_{i}$ in this permutation. Let $\mathcal{R}_{0}=\mathcal{R}$. Apply Lemma III.3 (with $R_{\pi^{-1}(1)} \in \mathcal{R}_{0}$ as the pusher) to get a core decomposition, denoted by $\mathcal{R}_{1}$, of $\mathcal{R}_{0}$. Now apply Lemma III.3 on $\mathcal{R}_{1}$ (with $\tilde{R}_{\pi^{-1}(2)} \in \mathcal{R}_{1}$ as the pusher) to get the set $\mathcal{R}_{2}$. Continuing iteratively by applying Lemma III.3 with the successive core of each $R_{\pi^{-1}(i)}$ in $\mathcal{R}_{i}$ as the pusher, we get the set $\tilde{\mathcal{R}}=\mathcal{R}_{n}$. See Figure 2 for an example.

We have the following crucial fact:

Claim III.6. If $v\left(\tilde{R}_{l}\right)$ has a vertex corresponding to $(v, i, j)$, then $(v, i, j) \in R_{l}$ and furthermore,

$$
\begin{cases}\max \left\{\pi_{i}, \pi_{j}\right\}<\min _{R_{m} \ni v} \pi_{m} & \text { if } l \neq i, j \\ \pi_{j}<\pi_{i} & \text { if } l=i \\ \pi_{i}<\pi_{j} & \text { if } l=j\end{cases}
$$




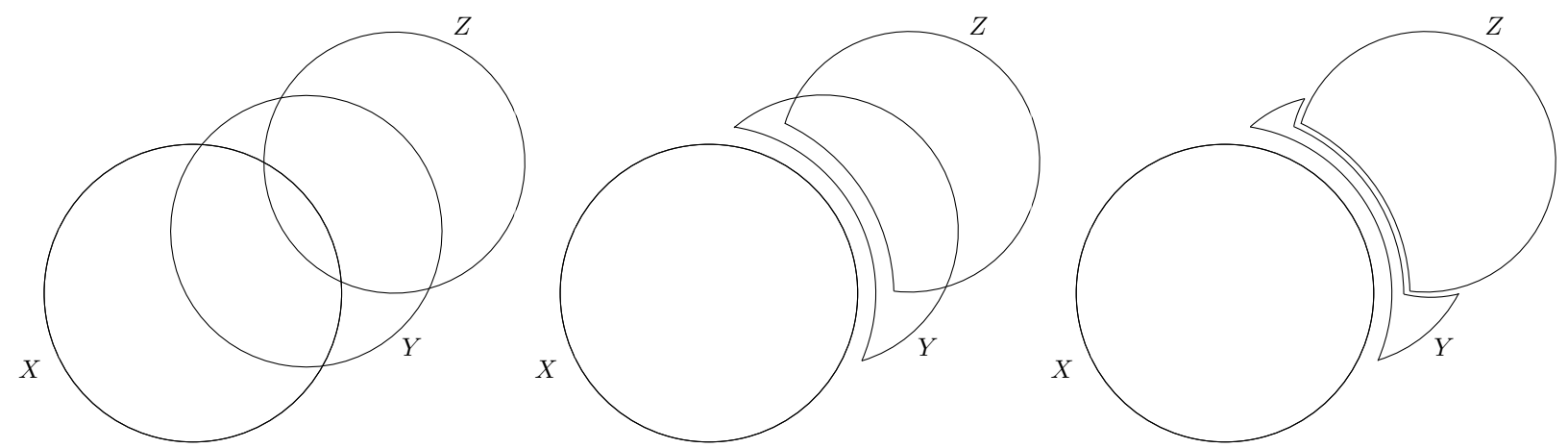

Figure 2: $X$ is the first pusher, $Z$ is the second pusher and $Y$ is the third pusher.

Proof: First consider the case when $l \neq i, j$. The proof relies on the observation that by the proof of Lemma III.3, if $v$ is a vertex in the arrangement of $\mathcal{R}$, and at any point a region containing $v$ in its interior is used as a pusher, then any $\tilde{R} \in \tilde{\mathcal{R}}$ cannot have a vertex that corresponds to $v$. Thus the only way a vertex corresponding to $(v, i, j)$ can be part of the boundary of any $\tilde{R}$ is if both the regions $R_{i}$ and $R_{j}$ occur earlier in $\pi$ than any of the regions containing $v$. For the case when $l=i$ (same for when $l=j$ ), the proof follows from the fact that if at any point there are two regions $X$ and $Y$ and $X$ is used as a pusher before $Y$, then the core of $X$ cannot have a vertex that corresponds to a vertex defined by $X$ and $Y$ (see Figure 2).

Then

$$
\begin{aligned}
\sum_{i}\left|v\left(\tilde{R}_{i}\right)\right| \cdot w_{i} & =\sum_{(v, i, j)} \sum_{l \text { s.t. } v \in v\left(\tilde{R}_{l}\right)} w_{l} \\
& =\sum_{(v, i, j)} \sum_{l \text { s.t. } v \in R_{l}} X_{(v, i, j, l)} \cdot w_{l}
\end{aligned}
$$

where the indicator variable $X_{(v, i, j, l)}=1$ iff $v \in v\left(\tilde{R}_{l}\right)$ (more precisely, the vertex corresponding to $v$ is in $v\left(\tilde{R}_{l}\right)$ ). Using the above Claim and Claim III.4, we calculate the expected value of the required bound:

$$
\begin{aligned}
& \sum_{i} E\left[\left|v\left(\tilde{R}_{i}\right)\right|\right] \cdot w_{i} \\
= & \sum_{(v, i, j)} \sum_{l \text { s.t. } v \in R_{l}} E\left[X_{(v, i, j, l)}\right] \cdot w_{l} \\
= & \sum_{(v, i, j)} \sum_{\substack{\text { s.t. } v \in R_{l} \\
=}} \operatorname{Pr}\left[v \in v\left(\tilde{R}_{l}\right)\right] \cdot w_{l} \\
& \sum_{(v, i, j)}\left(\operatorname{Pr}\left[v \in v\left(\tilde{R}_{i}\right)\right] w_{i}+\operatorname{Pr}\left[v \in v\left(\tilde{R}_{j}\right)\right] w_{j}\right. \\
& \left.+\sum_{\substack{i, j \neq l \\
v \in R_{l}}} \operatorname{Pr}\left[v \in v\left(\tilde{R}_{l}\right)\right] w_{l}\right)
\end{aligned}
$$

$$
\begin{aligned}
&=\sum_{(v, i, j)}( \frac{w_{j}}{w_{i}+w_{j}+d_{v}} \frac{w_{i}}{w_{i}+d_{v}} w_{i} \\
&+\frac{w_{i}}{w_{i}+w_{j}+d_{v}} \frac{w_{j}}{w_{j}+d_{v}} w_{j} \\
&+\sum_{\substack{i, j \neq l \\
v \in R_{l}}}\left(\frac{w_{j}}{w_{i}+w_{j}+d_{v}} \frac{w_{i}}{w_{i}+d_{v}}\right. \\
&\left.\left.+\frac{w_{i}}{w_{i}+w_{j}+d_{v}} \frac{w_{j}}{w_{j}+d_{v}}\right) w_{l}\right) \\
&=\sum_{(v, i, j)}\left(\left(\frac{w_{j}}{w_{i}+w_{j}+d_{v}} \frac{w_{i}}{w_{i}+d_{v}}\right)\right. \cdot\left(w_{i}+\sum_{R_{l} \ni v} w_{l}\right) \\
&+\left(\frac{w_{i}}{w_{i}+w_{j}+d_{v}} \frac{w_{j}}{w_{j}+d_{v}}\right) \\
&=2 \sum_{(v, i, j)} \frac{\left.\cdot\left(w_{j}+\sum_{R_{l} \ni v} w_{l}\right)\right)}{w_{i}+w_{j}+d_{v}} \\
&=2 \sum_{k} \sum_{2^{k} \leq d_{v}<2^{k+1}} \frac{w_{i} \cdot w_{j}}{w_{i}+w_{j}+d_{v}} \\
&=O(W \log W) .
\end{aligned}
$$

where the last inequality follows from Claim III.4.

We can now finish the proof of Theorem III.1.

Proof of Theorem III.1: We use Lemma III.5 to obtain a core $\tilde{Q}$ for each $Q \in \mathcal{Q}=\operatorname{Opt}(\mathcal{R}, P)$ and we assign to $\tilde{Q}$ the same weight as $Q$. Since the regions in $\mathcal{Q}$ cover $P$ their cores also cover $P^{5}$. As before, we denote the number of vertices in $\tilde{Q}$ by $|v(\tilde{Q})|$. By Lemma III.5, $\left.\sum_{Q \in \mathcal{Q}}|v(\tilde{Q})| w(Q)=O(w(\mathcal{Q}) \log w(\mathcal{Q}))\right)$. We set $\tau=C \cdot \frac{1}{\delta} \log w(\mathcal{Q})$ for some large enough constant $C$. Then by averaging, $\sum_{Q \in \mathcal{Q}:|v(\tilde{Q})|>\tau} w(Q)<\delta w(\mathcal{Q})$.

Let $\mathcal{Q}_{s}=\{Q \in \mathcal{Q}:|v(\tilde{Q})| \leq \tau\}$ and let $\tilde{\mathcal{Q}}_{s}=\{\tilde{Q}:$

\footnotetext{
${ }^{5}$ Since no point lies on the boundary of any of the regions, there is a suitable choice of $\beta$ so that using $\beta$-core decompositions, we do not miss any of the points.
} 
$\left.Q \in \mathcal{Q}_{s}\right\}$. The regions in $\tilde{\mathcal{Q}}_{s}$ are $\alpha \tau$-simple since they have at most $\tau$ sides and each of the sides is a portion of the boundary of a single $\alpha$-simple region in $\mathcal{R}$. These regions have a total weight of $w\left(\mathcal{Q}_{s}\right)$. Thus applying Theorem II.2, we get separator $\mathcal{C}$ so that the total weight of the regions of $\mathcal{Q}_{s}$ whose cores lie in interior $(\mathcal{C})$ (exterior $(\mathcal{C})$ ) is at most $\frac{2}{3} w\left(\mathcal{Q}_{s}\right)$. Since the total weight of the regions in $\mathcal{Q} \backslash \mathcal{Q}_{s}$ is at most $\delta w(\mathcal{Q})$, the total weight of all the cores that lie in interior $(\mathcal{C})($ exterior $(\mathcal{C}))$ is at most $\left(\frac{2}{3}+\delta\right) w(\mathcal{Q})$. Also, the total weight of the cores in $\mathcal{Q}_{s}$ that intersect $\mathcal{C}$ is at most $\delta w\left(\mathcal{Q}_{s}\right)$. Thus the total weight of all the cores in $\mathcal{Q}$ that intersect $\mathcal{C}$ is at most $2 \delta w(\mathcal{Q})$. The complexity of $\mathcal{C}$ is $O(\alpha \tau / \delta)=O\left(\frac{\alpha}{\delta^{2}} \log w(\mathcal{Q})\right)$, satisfying the fourth item in the statement of the theorem.

Let $\mathcal{Q}_{1}\left(\mathcal{Q}_{2}\right)$ be the set of regions whose cores are in interior $(\mathcal{C})($ exterior $(\mathcal{C}))$. Let $\mathcal{Q}_{3}=\mathcal{Q} \backslash\left\{\mathcal{Q}_{1} \cup \mathcal{Q}_{2}\right\}$. Observe that the cores of the regions in $\mathcal{Q}_{1} \cup \mathcal{Q}_{3}$ cover all the points in $P_{\text {in }}(\mathcal{C})$ and therefore the regions in $\mathcal{Q}_{1} \cup \mathcal{Q}_{3}$ themselves cover the points in $P_{\text {in }}(\mathcal{C})$. Similarly the regions in $Q_{2} \cup \mathcal{Q}_{3}$ cover the points in $P_{\text {ext }}(\mathcal{C})$. Therefore,

$$
\begin{aligned}
w\left(\operatorname{Opt}\left(\mathcal{R}, P_{i n}\right)\right) \leq w\left(\mathcal{Q}_{1} \cup \mathcal{Q}_{3}\right) & =w\left(\mathcal{Q}_{1}\right)+w\left(\mathcal{Q}_{3}\right) \\
& \leq\left(\frac{2}{3}+3 \delta\right) w(\mathcal{Q})
\end{aligned}
$$

This proves the first item in the statement of the theorem. The second item is proved analogously. For the third item, we combine the inequalities $w\left(\mathrm{OPT}\left(\mathcal{R}, P_{\text {in }}\right)\right) \leq w\left(\mathcal{Q}_{1}\right)+$ $w\left(\mathcal{Q}_{3}\right)$ and $w\left(\operatorname{Opt}\left(\mathcal{R}, P_{\text {ext }}\right)\right) \leq w\left(\mathcal{Q}_{2}\right)+w\left(\mathcal{Q}_{3}\right)$. We get

$$
\begin{array}{r}
w\left(\operatorname{Opt}\left(\mathcal{R}, P_{i n}\right)\right)+w\left(\operatorname{OPT}\left(\mathcal{R}, P_{\text {ext }}\right)\right) \\
\leq w\left(\mathcal{Q}_{1}\right)+w\left(\mathcal{Q}_{2}\right)+2 w\left(\mathcal{Q}_{3}\right) \\
\leq w(\mathcal{Q})+w\left(\mathcal{Q}_{3}\right) \leq(1+2 \delta) w(\mathcal{Q})
\end{array}
$$

That proves the third item.

Remark: The above QPTAS can be extended to work for more general regions called non-piercing regions or $r$ admissible regions. For this only Lemma III.3 needs to be extended to work for these regions. In this case, a region $R$ may intersect the boundary of pusher $X$ in more than one interval. To ensure that after pushing the new regions are still non-piercing, different gaps are required in different intervals for the same region $R$. This makes it technically more complicated.

\section{QPTAS FOR WEIGHTED HALFSPACES IN $\mathbb{R}^{3}$}

Let $\mathcal{H}=\left\{H_{1}, \cdots, H_{n}\right\}$ be a set of halfspaces in $\mathbb{R}^{3}$ where the halfspace $H_{i}$ has weight $w_{i} \geq 0$ and $W$ total weight. Let $P$ be a set of points in $\mathbb{R}^{3}$. Given $\mathcal{H}$ and $P$, we show that the problem of computing a subset of $\mathcal{H}$ of minimum weight whose union covers $P$ is QPT-partitionable, and then Lemma II.1 implies the QPTAS.

Consider the optimal solution OPT $=\operatorname{Opt}(\mathcal{H}, P)$ for the problem, and let $W$ be the total weight of the halfspaces in
Opt. For any halfspace $H$, define $\bar{H}$ to be other halfspace defined by its boundary $\partial H$ i.e., $\bar{H}=\operatorname{closure}\left(\mathbb{R}^{3} \backslash H\right)$. For any set of halfspaces $\mathcal{S}$, define $\overline{\mathcal{S}}=\{\bar{H}: H \in \mathcal{S}\}$.

Lemma IV.1. If $\bigcup_{H \in \mathrm{OPT}} H=\mathbb{R}^{3}$, then one can compute $\operatorname{Opт}(\mathcal{H}, P)$ in polynomial time.

Proof: If $\bigcup_{H \in \mathrm{OPT}} H=\mathbb{R}^{3}$, then by definition $\bigcap_{H \in \mathrm{OPT}} \bar{H}=\emptyset$. By Helly's theorem [15] applied to the set of convex regions in $\overline{\mathrm{OPT}}$, it follows that then there must be a subset $\mathrm{OPT}^{\prime} \subset$ OPT of at most 4 halfspaces such that $\bigcap_{H \in \mathrm{OPT}^{\prime}} \bar{H}=\emptyset$. In other words, OPT ${ }^{\prime}$ covers $\mathbb{R}^{3}$. As OPT was a minimal-weight set cover, it follows that $|\mathrm{OPT}| \leq 4$. By enumerating all 4-tuples of halfspaces in $\mathcal{H}$, one can compute the optimal set-cover in polynomial time.

From now on we assume that there is a point $o$ that does not lie in any of the halfspaces in OPT (say the origin). We will also assume without loss of generality that the intersection of halfspaces in $\overline{\text { OPT }}$ is a bounded polytope. This can be easily done by adding to the input four halfspaces with weight 0 which do not contain any of the points in $P$ whose complements intersect in a bounded simplex. These four dummy halfspaces can then be included in any optimal solution without affecting the weight of the solution. Note also that each halfspace $H \in$ OPT must be part of some facet (in fact, a unique facet) of this polytope; otherwise $H$ is contained in the union of OPT $\backslash\{H\}$, contradicting the set-cover minimiality of OPT.

We now define a core decomposition for the halfspaces in OPT that allows a cheap balanced polyhedral separator.

Consider the set system in which the base set are the halfspaces in OPT and subsets are defined by taking any segment $o x$ with one end-point at $o$ and taking the set of halfspaces whose boundaries intersect the segment. More formally, for any $x \in \mathbb{R}^{3}$, let $R_{x}=\{H \in$ OPT $: \partial H \cap o x \neq \emptyset\}$. We now define $\mathcal{R}$ as the set $\left\{R_{x}: x \in \mathbb{R}^{3}\right\}$. Consider the weighted set system $(\mathcal{H}, \mathcal{R})$, where the weight of $w(R)$ of any $R \in \mathcal{R}$ is the sum of the weights of the halfspaces in $R$.

Lemma IV.2. The VC-dimension of $(\mathcal{H}, \mathcal{R})$ is at most 3.

Proof: For two distinct points $x$ and $y$ that lie in the same cell of the arrangement of the halfspaces in OPT, $R_{x}=R_{y}$. So for any subset of OPT of size $k$, the number of induced subsets is at most the number of cells in an arrangement of these $k$ halfspaces, which is at most $\left(\begin{array}{l}k \\ 3\end{array}\right)+\left(\begin{array}{l}k \\ 2\end{array}\right)+\left(\begin{array}{l}k \\ 1\end{array}\right)+\left(\begin{array}{l}k \\ 0\end{array}\right)$. For $k=4$, this number is less than $2^{k}$, implying that no subset of size 4 is shattered. Thus the VC-dimension this set system is at most 3 .

Thus, by the $\epsilon$-net theorem [13], there is an $\epsilon$-net for this set system of size $O\left(\frac{1}{\epsilon} \log \frac{1}{\epsilon}\right)$. Let $N$ be an $\epsilon$-net for this set system for a value of $\epsilon$ to be fixed later. As before, we will assume that the intersection of halfspaces in $\bar{N}$ is a bounded polytope $\mathcal{P}$. This can be ensured by including in 
$N$ the dummy halfspaces.

For any set $S \subset \mathbb{R}^{3}$, define cone $(S)$ to be the set $\{\lambda x$ : $x \in S, \lambda \geq 0\}$. For any halfspace $H \in N$, we define the core of $H$ to be $\tilde{H}=$ cone $(f) \cap H$ where $f$ is the facet of $\mathcal{P}$ corresponding to $H$ i.e., the facet contained in $\partial H$. Note that each halfspace in $N$ (with the exception of dummy halfspaces) has a unique facet of $\mathcal{P}$ corresponding to it. For any halfspace $H \in \mathrm{OPT} \backslash N$, we defined the core as $\tilde{H}=$ $\mathcal{P} \cap H$. The core of each halfspace is clearly contained in the halfspace and the union of these cores is clearly the same as the union of the halfspaces in OPT.

We now assign a weight to each of the facets of $\mathcal{P}$ by distributing the weights of the halfspaces in OPT to the facets so that the total weight of the faces is the same as the total weight of the halfspaces. The weight of each halfspace in $N$ is assigned to facet corresponding it. For a halfspace in $H \in \mathrm{OPT} \backslash N$, we distribute its weight equally among all faces $f$ s.t. cone $(f)$ intersects the core $\tilde{H}$ of $H$.

The 1-skeleton of $\mathcal{P}$ is a planar graph $G$ and we have assigned weights to its faces. Let $n^{\prime}$ denote the number of vertices in this graph; note that $n^{\prime}=O(|N|)=O\left(\frac{1}{\epsilon} \log \frac{1}{\epsilon}\right)$. By [17], there exists in this graph a cycle separator $\mathcal{C}$ of size $O\left(\sqrt{n^{\prime}}\right)$ so that the total weight of the faces in the interior (exterior) of $\mathcal{C}$ is at most two thirds of the total weight. We show that the polytope $\hat{\mathcal{C}}=\operatorname{cone}(\mathcal{C})$ is the desired cheap balanced separator for the cores we have defined. $\hat{\mathcal{C}}$ splits $\mathbb{R}^{3}$ into two connected pieces whose closures we call the interior and the exterior of $\hat{\mathcal{C}}$. The choice is arbitrary. Note that interior $(\hat{\mathcal{C}}) \cap$ exterior $(\hat{\mathcal{C}})=\hat{C}$.

First note that for each core that lies in the interior (exterior), the weight of the corresponding halfspaces is distributed only among the faces of $\mathcal{P}$ lying in the interior (exterior) of $\hat{\mathcal{C}}$. Hence the total weight of all cores that lie in the interior (exterior) of $\hat{C}$ is at most two thirds of the total weight of all halfspaces.

We now need to bound the total weight of the cores that cross $\hat{\mathcal{C}}$. None of the cores of the halfspaces in $N$ cross $\hat{C}$. Consider a halfspace $H \in \mathrm{OPT} \backslash N$. Its core is defined as $H \cap \mathcal{P}$. If this core intersects $\hat{\mathcal{C}}$, then $H \cap \partial \mathcal{P}$ intersects $\mathcal{C}$. It follows that $H$ intersects an edge of $\mathcal{C}$ and thus must contain a vertex $v$ of $\mathcal{C}$. In other words, $\partial H$ intersects the segment $o v$. However, since $N$ is an $\epsilon$-net, for any vertex $v$, the total weight of halfspaces whose boundaries intersects $o v$ is at most $\epsilon W$. Since $\mathcal{C}$ has $O\left(\sqrt{n^{\prime}}\right)$ vertices, the total weight of all cores crossing $\hat{C}$ is $O\left(\sqrt{n^{\prime}} \cdot \epsilon W\right)$. We set $\epsilon=A \delta^{2} / \log \delta^{-2}$ for a suitable constant $A$ so that $\mathcal{C}$ has $O\left(\frac{1}{\delta} \log \frac{1}{\delta}\right)$ vertices and the total weight of cores intersecting is at most $\delta W$.

Finally, observe that the complexity of $\hat{\mathcal{C}}$ is determined by the complexity of $\mathcal{C}$, and the point $o$. The vertices of $\mathcal{C}$ are determined by intersections of 3 halfspaces of $\mathcal{H}$, and so there are $O\left(n^{3}\right)$ choices for each vertex of $\mathcal{C}$. To guess the point $o$, it suffices to guess the cell of the arrangement of $\mathcal{H}$ in which it lies (there are $O\left(n^{3}\right)$ such choices), and pick any point in that cell.

Remark: It may appear that the set cover problem for halfspaces may be reduced to the problem for pseudodisks using techniques used in [16]. Unfortunately, that does not work because (i) we are in the weighted setting and (ii) because we cannot tolerate the loss of a constant factor when looking for a $(1+\epsilon)$-approximation algorithm. It is also tempting to think that the technique used for halfspaces may be used for pseudodisks in the plane. That would mean taking an $\epsilon$-net $N$ for a suitable range space and then defining the core for each pseudodisks $R \notin N$ by removing from $R$ the portion of it covered by the union of pseudodisks in $N$. However, the problem in doing this is that the resulting cores may not be connected. This causes problems because if the cores are not connected then the cores not intersecting a separator curve $\mathcal{C}$ may still cover points in both interior $(\mathcal{C})$ and exterior $(\mathcal{C})$.

\section{LOWER-BOUNDS}

In this section we give examples of regions of low union complexity that are provably hard. Note that the problem is APX-hard even for a set $\mathcal{R}$ of $n$ regions in the plane of linear union complexity [11]. Furthermore:

Observation V.1. The problem of approximating minimumsize set-cover is:

1) APX-hard for a set $\mathcal{R}$ of $n$ 4-sided polygons in the plane of union complexity $O(n \alpha(n))$.

2) inapproximable within $o(\log s)$ factor for a set $\mathcal{R}$ of $n 4 s$-sided polygons in the plane of union complexity $n 2^{\alpha(n)^{O(s)}}$, for any integer $s>3$.

3) inapproximable within $o(\log d)$ factor for a set $\mathcal{R}$ of $n$ halfspaces in $\mathbb{R}^{d}$, for any integer $d>3$.

Proof: 1. Chan-Grant [4] showed that computing minimum size set-covers for objects defined by shadows of line-segments in the plane is APX-hard. By DavenportSchinzel sequences, the union complexity of $n$ line-segments in the plane is $O(n \alpha(n))$. These shadows can be 'closed off' without any further intersections to derive the 4 -sided polygons.

2. Trevisan [25] showed that computing minimum size setcovers for general set systems $\left(V,\left\{S_{1}, \ldots, S_{m}\right\}\right)$ where each $S_{i}$ has size $O(s)$ is inapproximable with factor $o(\log s)$ unless $P=N P$. These sets can be easily implemented using regions whose boundaries cross at most $O(s)$ times. To see this place a point corresponding to each vertex on the $x$-axis. Then for each set $S_{i}$ construct $x$-monotone curve $\gamma_{i}$ with $2 s+1$ horizontal segments and $2 s$ nearly vertical segments so that the points corresponding to the vertices in $S_{i}$ lie above $\gamma_{i}$ and all other points lies below $\gamma_{i}$. These 
curves can easily be drawn in such a way that any two of them intersect at most $O(s)$ times. Thus by bounds known on Davenport-Schinzel sequences, the lower envelope of the curves has complexity $O\left(n 2^{\alpha(n)^{O(s)}}\right)$. Thus if we consider the regions $R_{i}$ defined by the set of points above $\gamma_{i}$ we get a set of regions with small union complexity. These regions can be made bounded without increasing the union complexity.

3. There exist a set of points in $\mathbb{R}^{d}$ (points on the moment curve; see Matousek [15]) such that every $d / 2$-sized subset can be obtained by intersection with a halfspace. Thus a set-cover problem where every set has size at most $d / 2$ can be realized with halfspaces in $\mathbb{R}^{d}$, which together with the Trevisan bound [25] implies the lower-bound.

\section{CONCLUSION}

In this paper we demonstrated the versatility of separatorbased algorithmic design on a problem seemingly unrelated to the packing problems for which the separator had previously been successfully applied. Getting a polynomialtime approximation scheme for the set-cover problem for weighted pseudodisks in the plane and weighted halfspaces in $\mathbb{R}^{3}$ remains a very interesting open problem.

\section{ACKNOWLEDGMENT}

The authors would like to thank Janos Pach for many valuable discussions and encouragement. We also thank the reviewers for insightful feedback and comments.

\section{REFERENCES}

[1] A. Adamaszek and A. Wiese. Approximation schemes for maximum weight independent set of rectangles. In Proceedings of the 54th Annual IEEE Symposium on Foundations of Computer Science (FOCS), 2013.

[2] A. Adamaszek and A. Wiese. A QPTAS for maximum weight independent set of polygons with polylogarithmically many vertices. In SODA, 2014.

[3] Pankaj K. Agarwal and Jiangwei Pan. Near-linear algorithms for geometric hitting sets and set covers. In Symposium on Computational Geometry, 2014.

[4] Timothy M. Chan and Elyot Grant. Exact algorithms and APX-hardness results for geometric packing and covering problems. Comput. Geom., 47(2):112-124, 2014.

[5] Timothy M. Chan, Elyot Grant, Jochen Könemann, and Malcolm Sharpe. Weighted capacitated, priority, and geometric set cover via improved quasi-uniform sampling. In SODA, 2012.

[6] Victor Chepoi and Stefan Felsner. Approximating hitting sets of axis-parallel rectangles intersecting a monotone curve. Comput. Geom., 46(9):1036-1041, 2013.

[7] K. Clarkson and K. Varadarajan. Improved approximation algorithms for geometric set cover. Discrete Comput. Geom., 37:43-58, 2007.

[8] Gruia Călinescu, Ion I. Mandoiu, Peng-Jun Wan, and Alexander Z. Zelikovsky. Selecting forwarding neighbors in wireless ad hoc networks. Mob. Netw. Appl., 9(2):101-111, 2004.
[9] Thomas Erlebach and Erik Jan van Leeuwen. Approximating geometric coverage problems. In Proceedings of the nineteenth annual ACM-SIAM symposium on Discrete algorithms, SODA '08, 2008.

[10] Shashidhara K. Ganjugunte. Geometric Hitting Sets and Their Variants. PhD thesis, Duke University, 2011.

[11] Sariel Har-Peled. Being fat and friendly is not enough. CoRR, abs/0908.2369, 2009.

[12] Sariel Har-Peled. Quasi-polynomial time approximation scheme for sparse subsets of polygons. In Symposium on Computational Geometry, 2014.

[13] D. Haussler and E. Welzl. Epsilon-nets and simplex range queries. Discrete Comput. Geom., 2:127-151, 1987.

[14] Dorit S. Hochbaum and Wolfgang Maass. Approximation schemes for covering and packing problems in robotics and vlsi. In STACS, pages 55-62, 1984.

[15] J. Matousek. Lectures in Discrete Geometry. Springer-Verlag, New York, NY, 2002.

[16] J. Matousek, R. Seidel, and E. Welzl. How to net a lot with little: Small epsilon-nets for disks and halfspaces. In Proceedings of Symposium on Computational Geometry, pages 16-22, 1990.

[17] Gary L. Miller. Finding small simple cycle separators for 2-connected planar graphs. J. Comput. Syst. Sci., 32(3):265279, 1986.

[18] Nabil H. Mustafa. Approximation of Points: Combinatorics and Algorithms. Habilitation thesis, University of Paris-Est, 2013.

[19] Nabil H. Mustafa, Rajiv Raman, and Saurabh Ray. QPTAS for geometric set-cover problems via optimal separators. CoRR, abs/1403.0835, 2014.

[20] Nabil H. Mustafa and Saurabh Ray. Improved results on geometric hitting set problems. Discrete \& Computational Geometry, 44(4):883-895, 2010.

[21] S. Narayanappa and P. Vojtechovský. An improved approximation factor for the unit disk covering problem. In $C C C G$, 2006.

[22] Janos Pach and Micha Sharir. Combinatorial Geometry And Its Algorithmic Applications: The Alcala Lectures. Mathematical Surveys And Monographs. American Mathematical Society, 2009.

[23] Saurabh Ray. Weak and Strong Epsilon Nets for Geometric Range Spaces. Phd thesis, Saarland University, Germany, 2009.

[24] R. Raz and M. Safra. A sub-constant error-probability low-degree test, and a sub-constant error-probability PCP characterization of NP. In Proceedings of STOC, pages 475484, 1997.

[25] Luca Trevisan. Non-approximability results for optimization problems on bounded degree instances. In Proceedings of the Thirty-third Annual ACM Symposium on Theory of Computing, STOC '01, pages 453-461, 2001.

[26] K. Varadarajan. Weighted geometric set cover via quasi uniform sampling. In Proceedings of ACM Symposium on Theory of Computing, 2010. 